\title{
User-Friendly Website Design: A Combined Eye-Tracking Study
}

\author{
Julia Lamberz \\ Osnabrück University of Applied Science, Faculty of Management, Culture \\ and Technology (Lingen Campus), Germany \\ Thorsten Litfin \\ Osnabrück University of Applied Science, Faculty of Management, Culture \\ and Technology (Lingen Campus), Germany \\ Gunther Meeh-Bunse \\ Osnabrück University of Applied Science, Faculty of Management, Culture \\ and Technology (Lingen Campus), Germany \\ Özlem Teckert \\ Osnabrück University of Applied Science, Faculty of Management, Culture \\ and Technology (Lingen Campus), Germany
}

\section{Abstract}

A simple and intuitive operation - the so-called usability analysis of websites - is indispensable in modern times. In particular, drop-out rates can be applied as an indicator of existing usability problems. Eye-tracking is a proven method for the evaluation of the usability of websites. The aim of the study was to improve the usability of the website of an educational institute for trainees in the region as the target group. For this purpose, the use of an eye-tracking technology was combined with a survey of 30 trainees. The eye-tracking study was task-oriented: subjects were asked to inform themselves of particular course offers, and to register for the relevant courses. This procedure and the subsequent survey provided tangible indications of the search and surfing behaviour of the target group as well as their assessment of the usability of the website. This combination of methods allows the development of recommendations for optimizing the website with regard to its orientation, structure and level of comprehensibility.

Keywords: eye-tracking, usability evaluation, website usability JEL classification: M15, M31

Acknowledgments: Special thanks to Christina Böning, Tugba Demir, Vivien Heidemann, Nadine Wiedener and the Bildungswerk Grafschafter Wirtschaft

\section{Introduction}

Websites are characterized by a single structure that ideally addresses the requirements of their target groups. However, day-to-day experience of web surfers reveals that they have to struggle with misleading menus, hidden information and complicated ordering processes (Vogt, 2002). The economic success of websites depends on the expectations and desires of the target group, and is at the same time a critical factor for the success of the related enterprise (Mich et al., 2003). Hence, usability has a significant impact on the development of websites. Already in the 1990's Nielsen defined criteria determining the usability of websites. He sums up the relevant message for websites with little user-friendly content in a nutshell: "The 
first law of e-commerce is that if users cannot find the product, they cannot buy it either." (Nielsen, 2012). Furthermore, he emphasizes the relevance of a user-friendly web presence that should be tailored to the demand of a particular target group.

The success of a website depends on the requirements of their customers. In addition, web designers must consider the dependency of the usability on the task list of the users (Shackel, 2009). A task-based analysis enables providers to identify alignment patterns as well as usability issues in a way that users and their requirements are the center of a website usability evaluation (Ehmke et al., 2007).

The objective of this empirical study is to examine the usability of the website of an educational establishment including their main target group, "trainees". The focus was on their enrolment in various courses. For this reason, a task-based eye-tracking study was combined with a subsequent questionnaire. The following section discusses theoretically which criteria may be applied to the evaluation of websites. Furthermore, previous studies are reviewed for the appropriateness of eye-tracking approaches in combination with questionnaires for the analysis of usability problems. The goal of the eye-tracking study was to identify usability problems in terms of their effectiveness, efficiency and user satisfaction. For this reason the duration of each search request and the scan path of subjects on the initial website were analyzed, followed by a computer-based questionnaire. Therefore, the results of the duration measurements were compared to the results of the questionnaire.

\section{Usability und Web-Usability}

The literature provides numerous definitions for usability, invariably related to the usability of software systems. Usability is generally classified by means of various parameters defining the user-friendliness of software systems as a quality criterion (Speicher, 2015). Hereby, the international and widespread Norm EN ISO 9241-11 is applicable and describes the notion of "usability" in three dimensions (DIN EN ISO 9241-11, 2006):

- Effectiveness in solving problems;

- Efficiency in system handling;

- User satisfaction in relation to the software.

Effectiveness means a user achieves his or her goal entirely and accurately. Furthermore, a user is efficient if he or she reaches his or her goal with little effort. If these goals are achieved, then the user is not impaired during his or her activities and he or she is satisfied (Schweibenz et al., 2003). On the one hand the definition of DIN ISO 9241-11 assigns usability criteria to the software system (efficiency of operation) and the user (user satisfaction), respectively. On the other hand, the definition includes task fulfilment in an efficient way which is the responsibility of the user. All important aspects of the usability of websites are included in this definition. For this reason, the three criteria of the ISO norm served as the basis for this eyetracking study.

Yet, the question arises: what are the implications for the usability of web pages (subsequently referred to as 'web usability')? Numerous studies conclude that web usability and, at the same time, user satisfaction depend on the following points: 1) the representation of information on websites; 2) instant retrieval of information and clarity of site contents. At the same time, the focus is on heterogeneity of users in terms of their profile, their unique user situation and their goal (Schweibenz et al. 2003). 


\section{Eye-Tracking}

The analysis of scan paths with the eye-tracking approach is motivated in particular by the eye-mind hypothesis. This concept asserts that people process all perceived visual information. For this reason, the assumption is made that scan paths are closely related to human cognitive processes (Just et al., 1976). From a technical point of view, eye-tracking measures the individual scan paths of subjects. In doing so, a user looks at a stimulus. The retention time of an eye on a particular point is indicated by a visual fixation.

Volckmann et al. (2006) ascertained that the fixation duration influences the attention and information uptake of a user. A longer fixation duration implies more attention and a longer time period for information uptake of a user. The duration of a fixation within a particular location can be visualized with so-called heat maps. In this way, the elements with the longest fixation duration are indicated with red and elements with the shortest fixation duration with green. While looking at a stimulus, eyes do not focus on a single position for an extended time. Rather, they jump back and forth within a minute. These visual jumps among fixation locations are called 'sakkade'. These sakkades help users to orient themselves within a website. Hence, conclusions may be drawn about guidance devices in a website (Ehmke et al., 2007). Equipment for scan path recording determines the ocular alignment of a subject with the support of an infrared camera. In this way, gazing (views) can be recorded and analyzed. Contemporary technical equipment and the most recent image processing software enable scientists to video-record gazing in real time (Duchowski, 2007).

\section{Web-Usability in Eye-Tracking Studies}

The methodology of eye-tracking has been established as a support for web usability tests. Numerous studies demonstrate that various eye-tracking approaches such as time to first fixation on target, fixation duration and fixation length help to identify web usability problems (Ehmke et al., 2007). During one of the first task-based eyetracking studies Goldberg et al. (1999) detected a correlation between eye-tracking metrics and usability problems. As a consequence, eye-tracking metrics were modified in follow-up studies. As a result, these metrics enabled the scientists to identify usability problems (Byrne et al., 1999, Cowen et al. 1999, Goldberg et al. 2002).

The newly gained insights on the connection between eye-tracking metrics and usability problems emphasize the relevance of efficiency (which goal is achieved in what length of time?) and effectiveness (will the goal be achieved entirely and correctly?). In order to also detect how users perceive, comprehend and interpret fixated information, measured values (measured by technical equipment must be supplemented by survey- data (Cooke, 2006)). These surveys might include questions about the perception and assessment of websites in order to detect the level of satisfaction.

\section{Methodology}

The objective of the combined eye-tracking/survey study was to analyze the website of the educational institution, "Bildungswerk Grafschafter Wirtschaft", and, in particular the registration process for events. The goal was to detect whether the assigned tasks can be solved instantly, in straight-forward manner and to the satisfaction of users. For this reason, the following questions were pivotal for the empirical research: 
- Effectiveness: was the search process finalized successfully?

- Efficiency: was the search process finalized quickly and straight-forwardly?

- Satisfaction: was the search process perceived as convenient and structured? Effectiveness and efficiency were analyzed with the help of eye-tracking approaches. The analysis software of the eye-tracking system was applied to measure the duration of search requests (see Table 1). Furthermore, the same system analyzed the scan paths on the landing site in order to interpret both effectiveness and efficiency of the search request. For this reason, so-called areas of interest (AOI) were generated for the horizontal and left navigation bar as well as for the search box. These elements are believed to support search requests. The metric "time to first fixation" provides information about the scan path direction at the beginning of the search process. The stationary eye-tracking system, "Tobii X60 - 60 Hz", enabling the actimetry and analysis of individual gaze behavior was employed for the documentation of the study.

A computer-based questionnaire was applied as a supplementary method to elicit the subjective perception and appraisal of the search process. Subjects had to rate this process on a Likert scale ( 1 = fully applies to $5=$ does not apply). Yet, the data of gazing duration measurements are compared with responses in the satisfaction survey.

In order to assess the website of the educational institution "Bildungswerkes Grafschafter Wirtschaft", 30 subjects from the main target group, the trainees, were selected. The selection process was entirely random in collaboration with the vocational schools of the region who allowed the selection of the potential subjects during classes. None of the subjects was acquainted with the website prior to the test period of the eye-tracking study. The subjects were asked to undertake a search request for a particular course, where three courses were randomly allocated to the trainees. These courses are typical mandatory classes for trainees intending to undertake further training. They should reflect the three core offerings of the educational work. In the follow-up session, trainees were asked to complete a computer-based questionnaire in order to identify their satisfaction with the website while undertaking typical tasks.

Table 1

Tasks of an eye-tracking exercise

\begin{tabular}{|c|c|c|c|}
\hline Nb. & Task/search request & $\begin{array}{l}\text { Number of } \\
\text { subjects }\end{array}$ & Measured value \\
\hline 1 & $\begin{array}{l}\text { Course: "Industrial clerks - preparation for } \\
\text { final exams" }\end{array}$ & 10 & $\begin{array}{l}\text { Duration from } \\
\text { landing page to } \\
\text { target site }\end{array}$ \\
\hline 2 & Course: "English for commercial trainees" & 12 & $\begin{array}{l}\text { Duration from } \\
\text { landing page to } \\
\text { target site }\end{array}$ \\
\hline 3 & $\begin{array}{l}\text { Course: "Contemporary etiquettes in the } \\
\text { professional environment and day-to- } \\
\text { day life for trainees, } 2017 \text { " }\end{array}$ & 8 & $\begin{array}{l}\text { Duration from } \\
\text { landing page to } \\
\text { target site }\end{array}$ \\
\hline
\end{tabular}

Note: number of subjects per search request

Source: author's illustration 


\section{Results}

The applied methodology was successful in terms of reconstructing and analyzing the search and browsing behavior of the participants. All participants used the navigation boards of the landing page as a reference after a short orientation. This means the search behavior may be referred to as targeted.

In order to verify the effectiveness of search behavior the scientists examined how many subjects were able to fulfil their tasks successfully. As a result, 27 out of 30 subjects were able to successfully finalize the search process (s. Table 2). This complies with a $90 \%$ success rate. Three subjects did not find the course "etiquettes". A reason for this might be the fact that this course was not explicitly listed in the submenu of the top navigation bar as in the case of the other courses offered. In this way, an indication for locating that course was missing. In addition, the search box was not prominently located in the upper left corner of the site and less colorful with the consequence that none of the eight subjects who registered for the course "etiquettes" recognized the search box (Table 2). In the beginning of the search process, courses were searched for using two distinct paths on the landing page. The analysis of the gaze plots revealed that after a short orientation phase the first view landed either on the horizontal or the left navigation bar. The heat map (Figure 1) displays the intensity with which subjects looked at particular elements of the website. The analysis of this gazing behavior supported the results of the gaze plot. Both display a clear concentration of views on both of the navigation bars.

Figure 1

Comparison of the landing page and heat map for the search request "etiquettes"
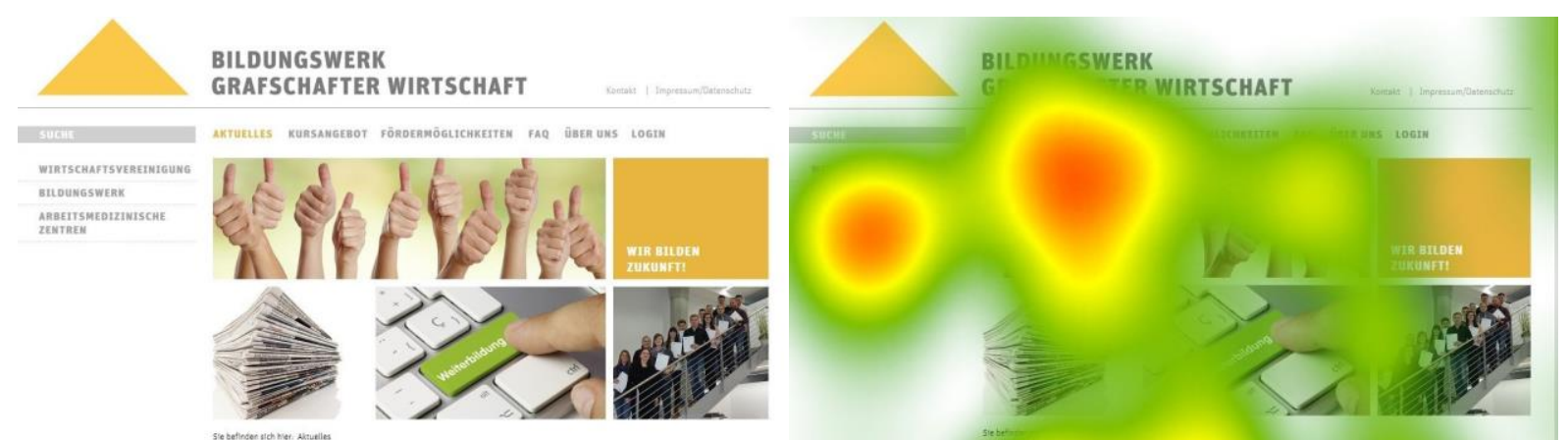

Note: Absolute duration is calculated by the duration of fixations, whereas the warmest color represents the highest value.

Source: author's illustration

The efficiency analysis requires the duration of task fulfilment (Table 2). Table 2

Duration of the search request and number of search interruptions

\begin{tabular}{lcccc}
\hline $\begin{array}{l}\text { Duration } \\
\text { in seconds }\end{array}$ & $\begin{array}{c}\text { Task 1: } \\
\text { Industrial clerks }\end{array}$ & $\begin{array}{c}\text { Task 2: } \\
\text { English }\end{array}$ & $\begin{array}{c}\text { Task 3: } \\
\text { Manners }\end{array}$ & All \\
\hline Minimum & 25.09 & 12.55 & 37.64 & 12.55 \\
Maximum & 112.21 & 82.94 & 47.16 & 112.21 \\
Mean & 64.02 & 40.22 & 43.54 & 49.65 \\
SD & 29.28 & 19.53 & 3.82 & 24.25 \\
\hline Cancellation & 0 & 0 & 3 & 3 \\
\hline
\end{tabular}

Source: author's calculation 
The average duration of each of the accomplished tasks was 49.7 seconds. Whereas three subjects were not able to find the course, the other subjects accomplished task 3 ("manners") surprisingly quickly in 43.5 seconds. Task 1 ("industrial clerk") was accomplished in the relatively slow time of $64.0 \mathrm{sec}$. This may be attributed to the individual queries during the exercise.

The detailed analysis of scan paths in relationship to the previously defined AOls on the landing page demonstrates that the time to first fixation of $2.3 \mathrm{sec}$ is the lowest (Table 3). However, the navigation box only contains general information about the school. No information about the task of finding a particular course offer can be found. The link "course offerings" is located in the horizontal navigation bar. However, this bar is recognized on average 0.5 seconds later than the left navigation box. It is remarkable that the time to first fixation in task 2 "English" and task 3 "etiquettes" is lowest ( $<1 \mathrm{sec}$ ) on the horizontal bar and other AOls are looked at later. Here, relevant information for the solution of the task was quickly identified. However, the search field was only recognized by six subjects after an average time of 28.3 seconds.

\section{Table 3}

Time to first fixation: navigation bars (left/horizontal) and search field per task

\begin{tabular}{|c|c|c|c|c|c|c|c|c|}
\hline \multirow[t]{2}{*}{$\begin{array}{l}\text { Time to first fixation (in } \\
\text { sec.) }\end{array}$} & \multicolumn{2}{|c|}{$\begin{array}{c}\text { Task 1: } \\
\text { Industrial clerks }\end{array}$} & \multicolumn{2}{|c|}{$\begin{array}{l}\text { Task 2: } \\
\text { English }\end{array}$} & \multicolumn{2}{|c|}{$\begin{array}{l}\text { Task 3: } \\
\text { Manners }\end{array}$} & \multicolumn{2}{|c|}{ All } \\
\hline & Mean & SD & Mean & SD & Mean & SD & Mean & SD \\
\hline $\begin{array}{l}\text { Navigation board left } \\
\text { "General information" }\end{array}$ & 1.99 & 2.79 & 2.17 & 2.36 & 3.72 & 3.41 & 2.27 & 2.62 \\
\hline $\begin{array}{l}\text { Navigation board } \\
\text { horizontal "Course } \\
\text { offer" }\end{array}$ & 6.29 & 15.20 & 0.96 & 0.73 & 0.52 & 0.06 & 2.75 & 9.02 \\
\hline Search field top left & 41.51 & 37.05 & 21.63 & 29.28 & n.a. & n.a. & 28.26 & 29.90 \\
\hline
\end{tabular}

Note: Duration in seconds "time to first fixation to AOl" of the landing page

Source: author's calculation

The results of the time to first fixation were compared to the results of the questionnaire in order to assess the satisfaction with the search process. The subjects evaluated the search requests as fast, straightforward and supportive for the navigation process. On the whole. the subjects were satisfied since they were able to accomplish the task effectively and efficiently. Only the search field was slightly difficult to find from the point of view of subjects. The assessments of the subjects was consistent with the results of the eye-tracking exercise.

\section{Table 4}

Appraisal and recognition of search requests

\begin{tabular}{lcc}
\hline Questions & All & SD \\
\hline The path was clearly recognizable. & 2.07 & 0.83 \\
I was able to orient myself quickly & 1.67 & 0.96 \\
The menu is clearly structured & 1.62 & 0.78 \\
The design is well arranged & 1.87 & 0.73 \\
I perceive the navigation as structured & 1.87 & 0.78 \\
I was able to find the search field instantly & 2.70 & 1.59 \\
Information browsing is time-consuming & 4.00 & 1.02 \\
Information browsing is complicated & 4.00 & 1.02 \\
\hline
\end{tabular}

Note: Likert scale where 1 = totally agree, 5 = totally disagree

Source: author's calculation 


\section{Discussion}

The combined eye-tracking/survey study provides insights into the usability of the website of the educational institution, "Bildungswerk Grafschafter Wirtschaft". The study revealed that measured durations of search requests cannot be viewed in isolation. Only after combining the analysis of scan paths on the landing page with a subsequent questionnaire was an indication found of the difficulties in locating the search field and the satisfaction of the target group with the search process. The search field should be highlighted in a better way and not placed in the upper left corner of the landing page. As in the case of many other websites the placement of the search field should be above the horizontal navigation bar.

In addition, the search behavior of trainees shows specific patterns indicting the importance of navigation bars and the designation of menu items. For this reason, important objects must conform to a logical structure and include intuitive key terms. The combined eye-tracking/questionnaire approach confirmed the results: the specific search requests were accomplished for the most part in an effective and efficient way, whereby subjects were satisfied.

\section{Conclusions}

The results of this eye-tracking study may serve as a basis for further studies of this kind. For example, eye-tracking metrics can be applied in order to improve the analysis of the search process, and develop recommendations for appropriate web design for specific target groups (Ehmke et al., 2007). This study merely developed one specific task and verified it. Further studies might include more tasks, for example, the evaluation of the site design and the involvement of more target groups. This approach has the potential to provide further results of relevance for web usability problems (Goldberg et al., 2002)

\section{References}

1. Byrne, M. D., Anderson, J. R., Douglass, S., Matessa, M. (1999), "Eye tracking the visual search of click-down menus", Proceedings of the SIGCHI conference on Human Factors in Computing Systems, ACM, 1999, pp. 402-409, available at: http://repository.cmu.edu/cgi/viewcontent.cgi? article=1022\&context=psychology (5 April 2017)

2. Cooke, L. (2006), "Is eye tracking the next step in usability testing?", International Professional Communication Conference, IEEE, pp. 236-242, available at: http://www.rose-hulman.edu/Users/faculty/williams/Public/PDF\%20Files/35.pdf (5 May 2017)

3. Cowen, L., Ball, J.L., Delin, J. (2001), "An eye movement analysis of webpage usability", available

http://citeseerx.ist.psu.edu/viewdoc/download?doi=10.1.1.106.4602\&rep=rep 1\&type=pdf (13 March 2017)

4. DIN EN ISO 9241-11 (2006), "Ergonomie der Mensch-System-Interaktion - Teil 11, Anforderungen an die Gebrauchstauglichkeit", Berlin, Beuth.

5. Duchowski, A. T. (2007), Eye Tracking Methodology - Theory and Practice, London, Springer.

6. Ehmke, C., Wilson, S. (2007) "Identifying web usability problems from eye-tracking data", Proceedings of the 21 st British $\mathrm{HCl}$ Group Annual Conference on People and Computers: $\mathrm{HCl}$... but not as we know it, Vol. 1, pp. 119-128.

7. Goldberg, J. H., Kotval, X. P. (1999), "Computer Interface Evaluation using Eye Movements: Methods and Constructs", International Journal of Industrial Ergonomics, Vol. 24, pp. 631-645. 
8. Goldberg, J. H., Stimson, M. J., Lewenstein, M., Scott, N., Wichansky, A. M. (2002), "Eye Tracking in Web Search Tasks: Design Implications", Eye Tracking Research \& Applications (ETRA) Symposium, ACM, pp. 51-58.

9. Just, M. A., Carpenter, P. A. (1976), "Eye fixations and cognitive processes." Cognitive psychology, Vol. 8 No. 4, pp. 441-480, available at: http://files.eric.ed.gov/fulltext/ED121119.pdf (29 March 2017)

10. Mich, L., Franch, M., Gaio, L. (2003), "Evaluating and designing web site quality", IEEE MultiMedia, Vol. 10 No. 1, pp. 34-43.

11. Nielsen, J. (2012), "Usability 101: Introduction to usability", available at: https://www.nngroup.com/articles/usability-101-introduction-to-usability/ (22 March 2017)

12. Schweibenz, W., Thissen, F. (2003), Qualität im Web: Benutzerfreundliche Webseiten durch Usability Evaluation, Berlin, Springer.

13. Shackel, B. (2009), "Usability - Context, framework, definition, design and evaluation", available at: http://sci-hub.cc/10.1016/j.intcom.2009.04.007 (22 March 2017).

14. Speicher, M. (2015), "What is usability?", available at: https://arxiv.org/pdf/1502.06792.pdf (22 March 2017)

15. Vogt, P. (2002), "Dem Surfer auf der Spur", c't- Magazin für Computertechnik, 14/2002, pp. 180.

16. Volckmann, J., Lippert, M. (2006), "Web Usability Testing", in: Arbeitspapiere WI, No. 1/2006, Eds.: Professur BWL - Wirtschaftsinformatik, Justus-Liebig-Universität, Gießen.

\section{About the authors}

Julia Lamberz is currently a Research Assistant at Osnabrveck University of Applied Sciences with a focus on marketing research. She received her Master's degree in Science Marketing from the Technische Universität Berlin. Author can be contacted at j.lamberz@hs-osnabrueck.de.

Thorsten Litfin is a Professor of Marketing, Service and Innovation Management at Osnabrück University of Applied Sciences. He received his PhD from the Institute of Innovation Management at Christian-Albrechts-University of Kiel. His research interests include product and pricing strategies for innovative products and services. Author can be contacted at t.litfin@hs-osnabrueck.de.

Gunter Meeh-Bunse is a Professor of Finance and Accounting at the University of Applied Sciences at Osnabrueck. He studied business administration at the University of Saarland and received his PhD from the University of German Armed Forces in Munich. His research interests include managerial accounting and corporate social responsibility. Author can be contacted at g.meeh-bunse@hs-osnabrueck.de.

Özlem Teckert is currently a Research Assistant at Osnabrück University of Applied Sciences with a focus on marketing research. She received her Master's degree in Economics and Laws from the University of Oldenburg and is currently a PhD candidate in the Department of Business Administration, Economics, and Law. Author can be contacted at o.teckert@hs-osnabrueck.de. 